John BAXENDALE, Priestley's England: J.B Priestley and English Culture

Manchester: Manchester University Press, 2007

\title{
Cécile Vallée
}

\section{(2) OpenEdition}

\section{Journals}

Édition électronique

URL : http://journals.openedition.org/rfcb/6086

DOI : $10.4000 /$ rfcb.6086

ISSN : 2429-4373

Éditeur

CRECIB - Centre de recherche et d'études en civilisation britannique

Édition imprimée

Date de publication : 1 avril 2008

ISSN : 0248-9015

\section{Référence électronique}

Cécile Vallée, «John BAXENDALE, Priestley's England: J.B Priestley and English Culture », Revue Française de Civilisation Britannique [En ligne], XIV-4 | 2008, mis en ligne le 01 avril 2008, consulté le 24 septembre 2020. URL : http://journals.openedition.org/rfcb/6086 ; DOI : https://doi.org/10.4000/rfcb. 6086

Ce document a été généré automatiquement le 24 septembre 2020

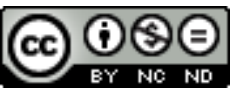

Revue française de civilisation britannique est mis à disposition selon les termes de la licence Creative Commons Attribution - Pas d'Utilisation Commerciale - Pas de Modification 4.0 International. 


\section{John BAXENDALE, Priestley's England: J.B Priestley and English Culture}

Manchester: Manchester University Press, 2007

Cécile Vallée

\section{RÉFÉRENCE}

John BAXENDALE, Priestley's England:J.B Priestley and English Culture, Manchester:

Manchester University Press, 2007, vii-213 p. ISBN 978-0-7190-7286-4.

1 Dans cet ouvrage, qui n'est ni une biographie ni une critique littéraire, John Baxendale se place du point de vue de l'historien. Son but est de rectifier l'image qui a été donnée de J.B Priestley. On a mal interprété sa vision de «l'Anglais », on lui a collé une fausse étiquette de "conservateur nostalgique», et surtout, on a sous-estimé son œuvre d'écrivain. Il ne compte pas dans l'histoire de la littérature parce que c'était un auteur au style désuet sans grande prétention intellectuelle, un auteur populaire - voire populiste - la popularité étant synonyme de médiocrité et de mercantilisme. John Baxendale entend prouver le contraire.

2 Il commence par retracer les origines de ces jugements négatifs, dans un premier chapitre, en replaçant l'auteur dans le conflit intellectuel et littéraire des années 20 et 30 entre les réalistes et les modernistes, ces « parvenus intellectuels » selon Priestley, dont il a lui-même vivement dénoncé les dérives. Pour Baxendale, Priestley est à bien des égards un auteur moderne, comme en témoignent sa popularité, sa participation dans le débat démocratique et son omniprésence sur la scène médiatique naissante. Il s'est attaché tout au long de sa carrière à remplir son devoir d'écrivain, à savoir de proposer à un public aussi large que possible une vision critique de la société contemporaine. Ce premier chapitre s'attache donc aussi à développer les débuts de la carrière de Priestley en tant que romancier, critique littéraire, membre de la «Book Society " journaliste, essayiste, homme de la radio écrivant aussi pour le cinéma. John Baxendale commence alors à passer en revue les premiers romans de Priestley en 
suivant une approche qui se veut chronologique, et donne au lecteur les bases de la pensée politique et littéraire de Priestley: celui-ci est foncièrement radical politiquement et socialement parlant, mais culturellement, dit-il, c'est un conservateur. Priestley estime en outre que l'objectif central d'un roman doit être de montrer l'homme dans la société dans laquelle il vit, une société qui doit être un personnage à part entière dans le roman et non plus seulement une trame de fond. Ces idées reviendront régulièrement tout au long de l'ouvrage.

3 Le deuxième chapitre, nous dit l'auteur, tente de démêler les nombreux fils de la vision du monde de Priestley. C'est un auteur plus complexe que l'on ne pense, dont les idées sont ancrées dans le radicalisme anglais. Il fait ressortir le «socialisme éthique » de l'auteur, plus proche du populisme que de la lutte des classes et montre comment Priestley s'est progressivement concentré sur l'observation de la société et l'expression débridée de ses opinions politiques. Il appartient à cette mouvance culturelle tournée vers l'investigation sociale, l'observation et la critique politique de la société. Baxendale voit en cette trajectoire un signe avant-coureur du rôle proéminent que Priestley allait jouer pendant la Seconde Guerre mondiale. Il explore alors les racines de sa critique sociale et politique, qu'il situe dans le Bradford d'avant la Première Guerre, sa ville natale, le berceau du mouvement travailliste et le «Bruddersford» de ses romans. Le « message » de l'écrivain commence à se préciser. Là encore, l'auteur oppose Priestley à ces intellectuels marxistes d'Oxford. En effet, Priestley n'a pas attendu la mode de la fin des années 30 pour se faire porte-parole du petit peuple, et John Baxendale nous le montrera tout au long du livre en passant au crible son œuvre et ses écrits. Ainsi, nous dit l'auteur, Priestley nous donne une image d'une Angleterre défigurée et noircie par l'appât du gain et le cynisme de quelques-uns. On l'accuse d'être un romantique et un nostalgique, mais il défend en fait la valeur de la vie et son idéalisme est d'ordre politique. Sans jamais renier ses origines du Nord de l'Angleterre, il condamne le capitalisme industriel, non pas au nom de la lutte des classes, qui ne fait pas partie de son discours politique, mais au nom de la démocratie et de l'esprit de communauté. Pour Baxendale, c'est cette vision de la société de Bradford sans classe que Priestley projette sur la nation tout entière. Il condamne le capitalisme parce que les valeurs sur lesquelles il repose sont à la fois immorales et inhumaines. Dans le conflit entre l'individualisme et le collectivisme, Priestley prône une société débarrassée des financiers et des politiciens, de l'oppression bureaucratique et du patriotisme mystificateur, nous explique l'auteur, qui met au centre des convictions de celui-ci la croyance en la force créatrice de chaque individu, quel qu'il soit. Contrairement aux marxistes qui, selon lui, déshumanisent les masses, Priestley défend l'épanouissement de l'individu.

4 Le lien entre les convictions de l'homme, son héritage culturel, sa vision de la société et ses idéaux est rendu limpide non seulement par une contextualisation socio-politique synthétique et éclairante, mais aussi par des citations multiples de Priestley lui-même, l'ensemble nous brossant un tableau complexe aux multiples facettes. Baxendale nous fait suivre la trajectoire de cet écrivain engagé au travers de ses écrits littéraires et journalistiques, dégageant ainsi les idées centrales de la pensée de Priestley, critique féroce des classes privilégiées coupées du peuple, des profiteurs, du déclin de la démocratie, du snobisme et du manque de créativité ambiant. Priestley est aussi un voyageur, un observateur qui transmet à ses lecteurs « ce qu'il voit, ce qu'il entend, ce 
qu'il ressent et ce qu'il pense ». En outre, si ses écrits sont ancrés dans le réel, ce réalisme est teinté de fantastique, de philosophie et de symbolique.

Après avoir ainsi imbibé le lecteur dans le monde politique, littéraire et philosophique de Priestley, John Baxendale explore, dans un troisième chapitre, la vision de l'Angleterre et des Anglais, sa conception de l'identité nationale, son patriotisme et son amour du petit peuple de l'Angleterre. Là encore, Baxendale réfute, parfois avec une grande vigueur, les jugements hâtifs et erronés portés sur Priestley qui révèlent une mauvaise connaissance de ses écrits. Loin d'être un défenseur du «mythe de l'Angleterre profonde ", (la plupart de ses romans se situent dans un cadre urbain) le réalisme de celui-ci est indissociable d'un idéalisme qui met en avant la relation entre l'homme et la nature. Le monde qui l'entoure est teinté de poésie, Priestley se fait l'avocat de la préservation d'une nature mise à mal par la modernité et sa prose n'est pas du tout au service d'un quelconque conservatisme rural, nous montre John Baxendale. Les préoccupations de Priestley concernent la société dans laquelle il vit, mais aussi les notions de temps, de mémoire et d'histoire.

6 Dans le chapitre 4, il nous donne toujours à voir cette Angleterre de Priestley, à comprendre son discours sur la modernité, l'industrialisation, l'américanisation de la société, (et sa vision de l'Amérique), mais aussi sur le cinéma, la presse et la radio.

7 Baxendale consacre bien sûr un chapitre sur le rôle de Priestley pendant la Seconde Guerre Mondiale qui l'a fait entrer dans l'Histoire. Porte-parole du « petit peuple » et de la reconstruction, ses «post-scriptum » du dimanche sur les ondes de la BBC pendant l'été 1940 sont devenus inséparables des discours de Winston Churchill à la même époque. Son énergie productive pendant les années de guerre est incroyable, puisqu'il n'écrit pas moins de 4 romans et 5 pièces de théâtre, publie de nombreux articles, écrit pour le cinéma et parle à la radio non seulement à ses compatriotes, mais aussi sur les ondes internationales, où il fait 39 allocutions à l'intention des Américains rien que pendant l'été 1940. En quelques pages, Baxendale nous montre avec justesse et précision combien Priestley excellait dans cet exercice d'équilibriste entre propagande officielle en accord avec les besoins du moment et critique politique à peine voilée des classes privilégiées, et nous expose avec brio les idées maîtresses des ces allocutions légendaires. Suit très logiquement (mais de façon un peu répétitive) un développement de la pensée politique de Priestley, son "socialisme utopique» né de ses préoccupations humanistes qui mettent l'individu au cœur de la société et affirme la dimension spirituelle de tout homme. La vérité, la beauté du monde, la magie de l'imagination, la part du divin en l'homme sont d'autres thèmes chers à Priestley. Baxendale analyse donc surtout la philosophie politique de Priestley, mais nous dévoile aussi la richesse et la poésie de son écriture.

8 Dans le dernier chapitre, l'auteur revient sur le rôle essentiel de la propagande dans l'élaboration de la mythologie dominante dans la Grande-Bretagne de l'après-guerre. Il suit son fil chronologique, tout en éclairant par des retours en arrière l'évolution de la pensée de J.B Priestley. L'après-guerre est marqué par la même prolixité (16 romans et autant de pièces de théâtre avec une variété de genre et de ton extraordinaire) et une critique toujours aussi énergique de la société et de la politique. Priestley, nous dit étonnamment John Baxendale, devient dans les années soixante un représentant de la contre-culture. Bien que socialiste, il continue de déplorer l'absence de véritable renouveau de la société anglaise : l'élan collectif est passé et retombé, la vision noble d'un objectif commun a disparu. Or, Priestley défend toujours l'idée d'une communauté 
créatrice qui ne s'embarrasserait pas de la bureaucratie ou de la culture par trop sérieuse prônée par le gouvernement travailliste. Et Baxendale de continuer à promener le lecteur dans l'univers culturel et politique de Priestley, son opposition à la centralisation du pouvoir et aux mécanismes bureaucratiques de l'état qui empêchent la démocratie locale et les impulsions créatrices du peuple de s'exprimer.

Observateur et commentateur infatigable de la société, comme nous le montre si bien John Baxendale, Priestley condamne aussi le nouveau système économique et socioculturel (qu'il qualifie d'un mot nouveau, «Admass ») qui aliène le peuple placé sous le joug du capitalisme et de la communication de masse, aveuglé par la publicité et le consumérisme qui détournent leurs rêves et leur idéalisme. Mais ce rejet de la civilisation de l'image et du spectacle, comme celui de la bureaucratie et de l'immobilisme gouvernemental (sans parler de la bombe atomique) ne doit pas nous faire oublier que Priestley était aussi un éternel optimiste. Ni un "edwardien nostalgique » ni même un homme tourné vers le passé, mais un visionnaire qui voulait réformer la société non par le haut, mais en donnant la parole au peuple, un idéaliste qui n'a jamais abandonné l'espoir d'une société où la vie de la communauté serait l'expression de la vie intérieure de l'individu réconcilié avec lui-même.

Tout ceci est admirablement expliqué et commenté, et un des grands mérites de cet ouvrage dense et exigeant réside dans le fait que Baxendale met en lumière les multiples thèmes chers à Priestley et fait voler en éclat les clichés et autres opinions fausses le concernant. La mise en perspective historique, sociale et politique nous aide à suivre les multiples allées et venues de l'auteur dans l'œuvre complexe et volumineuse de Priestley. Tout en retraçant l'atmosphère et l'histoire culturelle de plusieurs décennies, John Baxendale nous fait donc (re)découvrir l'univers de cet écrivain humaniste et philosophe d'une grande modernité dont l'idéal de société est loin d'avoir perdu de son actualité.

\section{AUTEURS}

CÉCILE VALLÉE

Université de Rouen 